\title{
Inhomogeneities in the Freeze-Out of Relativistic Heavy-Ion Collisions
}

\author{
Detlef Zschiesche and Licinio Portugal \\ Instituto de Física, Universidade Federal do Rio de Janeiro \\ C.P. 68528, Rio de Janeiro, RJ 21941-972, Brazil
}

Received on 6 November, 2006; revised version on 12 February, 2007

\begin{abstract}
An inhomogeneous decoupling surface of hadrons produced in relativistic heavy-ion collisions may occur, if the expanding hot and dense matter passes through a first order phase transition. We show that due to the non-linear dependence of the particle densities on the temperature and baryon-chemical potential such inhomogeneities should be visible even in the integrated, inclusive abundances. We analyze experimental data from $\mathrm{Pb}+\mathrm{Pb}$ collisions at CERN-SPS and $\mathrm{Au}+\mathrm{Au}$ collisions at BNL-RHIC to determine the amplitude of inhomogeneities and the role of local and global strangeness neutrality.
\end{abstract}

Keywords: Particle abundaces in heavy ion collisions; Inhomogeneous chemical freeze-out; Local and global strangeness neutrality

It is expected that at sufficiently high energies, a transient state of deconfined matter with broken $Z(3)$ center symmetry and/or with (approximately) restored chiral symmetry is produced in collisions of heavy nuclei. Lattice QCD simulations [1] indicate that a second-order critical point exists, which was also predicted by effective chiral Lagrangians [2]; present estimates locate it at $T \approx 160 \mathrm{MeV}, \mu_{B} \approx 360 \mathrm{MeV}$. This point, where the $\sigma$-field is massless, is commonly assumed to be the endpoint of a line of first-order phase transitions in the $\left(\mu_{B}, T\right)$ plane. To detect that endpoint, it is hoped that by varying the beam energy, for example, one can "switch" between the regimes of first-order phase transition and cross over, respectively. If the particles decouple shortly after the expansion trajectory crosses the line of first order transitions one would expect a rather inhomogeneous (energy) density distribution on the freeze-out surface [3, 4] (similar, say, to the CMB photon decoupling surface observed by WMAP [5]). On the other hand, if the low-temperature and high-temperature regimes are smoothly connected, pressure gradients tend to wash out density inhomogeneities. Thus, we investigate the properties of an inhomogeneous fireball at (chemical) decoupling. Note that if the scale of these inhomogeneities is much smaller than the decoupling volume then they can not be resolved individually, nor will they give rise to large event-by-event fluctuations. Because of the nonlinear dependence of the hadron densities on $T$ and $\mu_{B}$, they should nevertheless reflect in the average abundances.

Our basic assumption is that as the fireball expands and cools, at some stage the abundances of hadrons "freeze", keeping memory of the last instant of chemical equilibrium. This stage is refered to as chemical freeze-out. By definition, only processes that conserve particle number for each species individually, or decays of unstable particles may occur later on.

The simplest model is to treat the gas of hadrons within the grand canonical ensemble, assuming a homogeneous decoupling volume. The abundances are then determined by two parameters, the temperature $T$ and the baryonic chemical potential $\mu_{B}$; the chemical potential for strangeness is fixed by the condition for overall strangeness neutrality. Fits of hadronic ratios were performed extensively $[6,7]$ within this model, sometimes also including a strangeness $\left(\gamma_{s}\right)$ or light quark $\left(\gamma_{q}\right)$ supression factor $[8,9]$ or interactions with the chiral condensate [10].

In [11] we analyzed the experimental data on relative abundances of hadrons with respect to the presence of inhomogeneities on the decoupling surface. To that end we proposed a very simple and rather schematic extension of the common grand canonical freeze-out model, i.e. a superposition of such ensembles with different temperatures and baryonchemical potentials. Each ensemble is supposed to describe the local freeze-out on the scale of the correlation length $\sim 1 / T \sim 1-2 \mathrm{fm}$. Even if freeze-out occurs near the critical point, the correlation length of the chiral condensate is bound from above by finite size and finite time effects, effectively resulting in similar numbers [12]. On the other hand, for small chemical potential, far from the region where the $\sigma$-field is critical, the relevant scale might be set by the correlation length for Polyakov loops, which is of comparable magnitude [13]. The entire decoupling surface contains many such "domains", even if a cut on mid-rapidity is performed. We therefore expect that the distributions of temperature and chemical potential are approximately Gaussian [14]. Besides simplicity, another goal of the analysis is to avoid reference to a particular dynamical model for the formation or for the distribution of density perturbations. In fact, we presently aim merely at checking whether any statistically significant signal for the presence of inhomogeneities is found, and here especially, what influence local or global strangeness neutrality, respectively, have. More sophisticated dynamical models could be employed in the future to understand the evolution of inhomogeneities from their possible formation in a phase transition until decoupling. For simplicity, and for lack of an obvious motivation for assuming otherwise, we shall take $P\left(T, \mu_{B}\right)$ to factorize into a distribution for $T$, times one for $\mu_{B}$. These distributions could, in principle, be obtained from the real-time evolution of the phase transition $[3,4]$. The densities of strange particles depend also on the strangeness-chemical potential $\mu_{S}$, which we determined in [11] by imposing local strangeness neutrality. However, the effect of independent fluctuations of $\mu_{S}$ should also be looked at, in particular for collisions at low and intermediate energies $\left(\sqrt{ } s_{N N} \lesssim 15 \mathrm{GeV}\right)$. This may help to reproduce the $\bar{\Lambda}$ to $\bar{p}$ ratio, which was found 
to be larger than one [16] and the $K^{+} / \pi^{+}$enhancement around $E_{\mathrm{Lab}} / A=30 \mathrm{GeV}$ [17]. Then the hadron abundances depend on six parameters: the arithmetic means of the temperatures and chemical potentials of all domains, $\bar{T}, \bar{\mu}_{B}$ and $\bar{\mu}_{S}$, and the widths of their Gaussian distributions, $\delta T, \delta \mu_{B}$ and $\delta \mu_{S}$. They read:

$$
\begin{aligned}
& \bar{\rho}_{i}\left(\bar{T}, \bar{\mu}_{B}, \bar{\mu}_{S}, \delta T, \delta \mu_{B}, \delta \mu_{S}\right)=\int_{0}^{\infty} d T P(T ; \bar{T}, \delta T) \\
& \int_{-\infty}^{\infty} d \mu_{B} P\left(\mu_{B} ; \bar{\mu}_{B}, \delta \mu_{B}\right) \int_{-\infty}^{\infty} d \mu_{S} P\left(\mu_{S} ; \bar{\mu}_{S}, \delta \mu_{S}\right) \rho_{i}\left(T, \mu_{B}, \mu_{S}\right)
\end{aligned}
$$

with $\rho_{i}\left(T, \mu_{B}, \mu_{S}\right)$ the actual "local" density of species $i$, and with $P(x ; \bar{x}, \delta x) \sim \exp \left[-(x-\bar{x})^{2} / 2 \delta x^{2}\right]$ the distribution of temperatures and chemical potentials within the decoupling three-volume (the proportionality constants normalize the distributions over the intervals where they are defined). In addition, strangeness conservation enters now as a global constraint for the mean of the strange chemical potential $\bar{\mu}_{S}$ :

$$
\sum_{i} \bar{\rho}_{i}\left(\bar{T}, \bar{\mu}_{B}, \bar{\mu}_{S}, \delta T, \delta \mu_{B}, \delta \mu_{S}\right)\left(n_{s}^{i}-n_{\bar{s}}^{i}\right)=0
$$

with $n_{s}^{i}, n_{\bar{s}}^{i}$ the number of strange and anti-strange quarks of hadron species $i$, respectively. In the limit $\delta T, \delta \mu_{B}, \delta \mu_{S} \rightarrow 0$ the Gaussian distributions are replaced by $\delta$-functions and the conventional homogeneous freeze-out scenario is recovered: $\bar{\rho}_{i}\left(\bar{T}, \bar{\mu}_{B}, \bar{\mu}_{S}, 0,0,0\right)=\rho_{i}\left(\bar{T}, \bar{\mu}_{B}, \bar{\mu}_{S}\right)$ and the corresponding strangeness neutrality condition fixing $\bar{\mu}_{S}$. In other words, in that limit the average densities are uniquely determined by the first moments of $T$ and $\mu_{B}$. For the present analysis we set the width of the distribution for the strange chemical potential equal to zero, $\delta \mu_{S}=0$. Since eq.(2) only ensures global strangeness neutrality, this still offers the possibility of finite net strangeness values in individual domains, in contrast to our former analysis, where we fixed $\mu_{S}$ by $f_{s}=0$ locally. With setting $\delta \mu_{S}=0$ and the global constraint for $\overline{\mu_{S}}$, the densities again are a function of four parameters: $\bar{T}, \bar{\mu}_{B}, \delta T$ and $\delta \mu_{B}$. The densities $\rho_{i}\left(T, \mu_{B}, \mu_{S}\right)$ are computed in the ideal gas approximation, supplemented by an "excluded volume" correction:

$$
\rho_{i}\left(T, \mu_{B}, \mu_{S}\right)=\frac{\rho_{i}^{\mathrm{id}-\mathrm{gas}}\left(T, \mu_{B}, \mu_{S}\right)}{1+v_{i} \sum_{j} \rho_{j}^{\mathrm{id}-\mathrm{gas}}},
$$

where $v_{i}$ denotes the volume occupied by a hadron of species $i$ with $v=\frac{4}{3} \pi R_{0}{ }^{3}$ with $R_{0}=0.3 \mathrm{fm}$ for all species [15].

To determine the four parameters of the model we minimize

$$
\chi^{2}=\sum_{i}\left(r_{i}^{\text {exp }}-r_{i}^{\text {model }}\right)^{2} / \sigma_{i}^{2}
$$

in the space of $\bar{T}, \bar{\mu}_{B}, \delta T$, and $\delta \mu_{B}$. That is, we obtain leastsquare estimates for the parameters, assuming that they are independent. In (4), $r_{i}^{\text {exp }}$ and $r_{i}^{\text {model }}$ denote the experimentally measured and the calculated particle ratios, respectively, and $\sigma_{i}^{2}$ is set by the uncertainty of the measurement. Wherever available, we sum systematic and statistical errors in quadrature.

The data used in our analysis are the particle multiplicities measured by the NA49 collaboration for central $\mathrm{Pb}+\mathrm{Pb}$ collisions at beam energy $E_{\mathrm{Lab}} / A=20,30,40,80$ and $158 \mathrm{GeV}$ [17], and those measured by STAR for central $\mathrm{Au}+\mathrm{Au}$ collisions at BNL-RHIC, ref. [18, 19] (for individual references see [11] ref [27] and [28]). At RHIC energies, we analyze the midrapidity data; at SPS energies, we restrict ourselves to the $4 \pi$ solid angle data by NA49 in order to avoid biases arising from differing acceptance windows of various experiments. Where possible, we have opted for the least bias by choosing $r_{i}^{\text {exp }}=N_{i}^{\text {exp }} / N_{\pi}^{\text {exp }}$, i.e. the multiplicity of species $i$ relative to that of pions (for details see [11]). Where appropriate, feeding from strong and electromagnetic decays has been included in $r_{i}^{\text {model }}$ by replacing $\rho_{i} \rightarrow \rho_{i}+B_{i j} \rho_{j}$, for details about the decay table and the included resonances see [11].

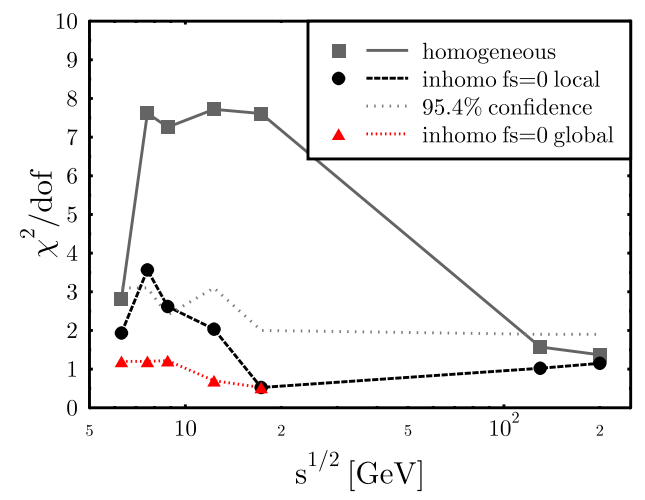

FIG. 1: $\chi^{2} / d o f$ versus $\sqrt{ } s_{N N}$ for the homogeneous $(\delta T=\delta \mu=0$, squares) and the inhomogeneous fit ( $\delta T$ and $\delta \mu$ free parameters). Circles denote the case of local strangeness neutrality, while triangles represent the global strangeness neutrality case. The lines are meant to guide the eye. Furthermore, the $\chi^{2} /$ dof corresponding to the $95.4 \%$ confidence interval is shown by the dotted line.

Fig. 1 shows the minimal $\chi^{2}$ per degree of freedom (number of data points minus the number of parameters) for the homogeneous approach and the inhomogeneous approach with local or global strangeness neutrality, respectively. Note that the $\chi^{2}$-values for the homogeneous model are in general agree-

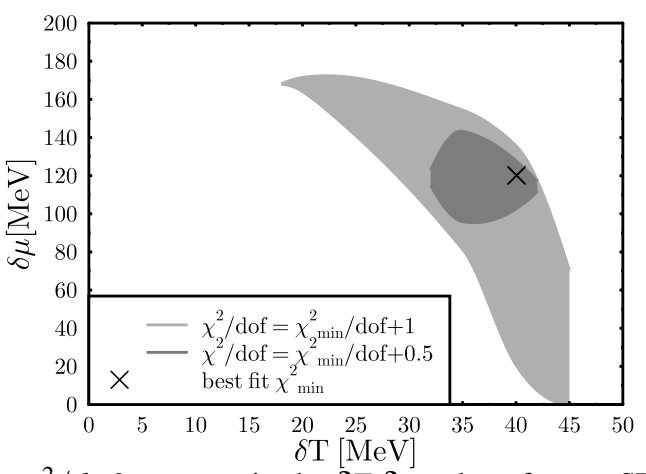

FIG. 2: $\chi^{2} / d o f$ contours in the $\delta T, \delta \mu_{B}$ plane for top SPS energy $\left(E_{\mathrm{Lab}}=158 \mathrm{GeV}\right)$ in the inhomogeneous freeze-out model with global strangeness neutrality. The other two parameters $\left(\bar{T}, \bar{\mu}_{B}\right)$ are allowed to vary freely. The $\chi^{2} /$ dof minimum is indicated by the cross. For details on the obtained $T, \mu_{B}$ freeze-out values see [11]. 
ment with the analysis done in [6] and other data from the literature $[7,8,19]$. As already shown in [11], for intermediate $S P S$ energies, $E_{\mathrm{Lab}} / A \simeq 30-160 \mathrm{GeV}, \chi^{2} /$ dof is considerably smaller for the inhomogeneous freeze-out surface than for the homogeneous case, which is far outside the $95.4 \%$ confidence interval [21]. On the other hand, at $E_{\mathrm{Lab}} / A=20 \mathrm{GeV}$ and especially at RHIC energies, the inhomogeneous case with local strangeness neutrality and the homogenous model give similar $\chi^{2} /$ dof. However, between 20 and $80 \mathrm{GeV}$ the $\chi^{2} / d o f$ values for the inhomogenous approach with local strangeness neutrality are still rather large (between 2 and 4). In contrast, the inhomogenoues model with globally vanishing net-strangeness gives $\chi^{2} /$ dof $\approx 1$ for $E_{\mathrm{Lab}} / A \simeq 20-160 \mathrm{GeV}$. It is important to note that this result is not due to introducing an additional parameter, but just due to allowing for domains of finite strangeness with global strangeness neutrality. Calculations for RHIC energies with global strangeness conservation are under way, but due to the corresponding small baryon chemical potentials at these high energies no considerable effect is expected. Thus, the inhomogeneous model gives a very satisfactory description $\left(\chi^{2} / d o f \approx 1\right)$ of the experimental data for particle abundance ratios from lowest SPS energies up to highest RHIC energies.

At RHIC the inhomogeneous model does not provide a statistically significant improvement of the description of the measured particle ratios compared to a homogeneous ap- proach. Thus, the assumption of a nearly homogeneous decoupling surface can not be rejected there. On the other hand, the considerable improvement of the description of the data for $E_{\mathrm{Lab}} / A \simeq 30-160 \mathrm{GeV}$ indicates that at intermediate and high SPS energies, the experimental data favor an inhomogeneous freeze-out surface. For the SPS $20 \mathrm{GeV}$ data the situation is not clear: there is certainly a reduction of the $\chi^{2} / d o f$ in the inhomogeneous approach, but the homogeneous model already gives a reasonable value. Here more experimental data are certainly necessary. The improved description of the experimental data when considering globally vanishing netstrangeness is because it allows for an increased production of strange and anti-strange particles in certain regions without a local constraint on the strange chemical potential.

To further illustrate the significance of inhomogeneities, we show contours of $\chi^{2} / d o f$ in the plane of $\delta T, \delta \mu_{B}$ in Fig. 2 for the case of global strangeness conservation at SPS 158. Here, $\bar{T}$ and $\bar{\mu}_{B}$ were allowed to vary freely such as to minimize $\chi^{2}$ at each point. The $\chi^{2}$ determines the $\delta \mu_{B}$ quite accurately, favoring relatively large finite values. For $\delta T$, values different from zero are strongly favored, corresponding to a very inhomogeneous decoupling surface. In contrast, at RHIC energy, we found $\chi^{2}$ to be very flat in both directions.

\section{Acknowledgements:}

We thank A. Dumitru for collaboration. The work was supported partially by CAPES and CNPq.
[1] Z. Fodor and S. D. Katz, JHEP 0404, 050 (2004).

[2] M. Stephanov, K. Rajagopal, and E. V. Shuryak, Phys. Rev. Lett. 81, 4816 (1998).

[3] D. Bower and S. Gavin, Phys. Rev. C 64, 051902 (2001).

[4] K. Paech and A. Dumitru, Phys. Lett. B 623, 200 (2005); K. Paech, H. Stöcker, and A. Dumitru, Phys. Rev. C 68, 044907 (2003); O. Scavenius, A. Dumitru, and A. D. Jackson, arXiv:hep-ph/0103219, Figs. 5,6.

[5] http://map.gsfc.nasa.gov/m_mm.html

[6] A. Andronic, P. Braun-Munzinger, and J. Stachel, Nucl. Phys. A 772, 167 (2006) [arXiv:nucl-th/0511071]

[7] see for example K. Redlich, J. Cleymans, H. Oeschler, and A. Tounsi, Acta Phys. Polon. B 33, 1609 (2002); P. BraunMunzinger, K. Redlich, and J. Stachel, arXiv:nucl-th/0304013; M. Michalec, arXiv:nucl-th/0112044; and references therein.

[8] J. Rafelski, J. Letessier, and A. Tounsi, Acta Phys. Polon. B 27, 1037 (1996); F. Becattini, M. Gazdzicki, A. Keranen, J. Manninen, and R. Stock, Phys. Rev. C 69, 024905 (2004);

[9] F. Becattini, J. Manninen, and M. Gazdzicki, arXiv:hep$\mathrm{ph} / 0511092$.

[10] D. Zschiesche, S. Schramm, J. Schaffner-Bielich, H. Stöcker, and W. Greiner, Phys. Lett. B 547, 7 (2002); W. Florkowski, W. Broniowski, and M. Michalec, Acta Phys. Polon. B 33, 761 (2002) ; D. Zschiesche, G. Zeeb, K. Paech, H. Stöcker, and S. Schramm, J. Phys. G 30, S381 (2004);

[11] A. Dumitru, L. Portugal, and D. Zschiesche, Phys. Rev. C 73, 024902 (2006) [arXiv:nucl-th/0511084].

[12] B. Berdnikov and K. Rajagopal, Phys. Rev. D 61, 105017 (2000); K. Paech, Eur. Phys. J. C 33, S627 (2004).

[13] A. Dumitru and R. D. Pisarski, Phys. Lett. B 504, 282 (2001); Nucl. Phys. A 698, 444 (2002).
[14] The investigation of other distributions and also the application of the "superstatistics" approach [23] are under way.

[15] P. Braun-Munzinger, I. Heppe, and J. Stachel, Phys. Lett. B 465, 15 (1999)

[16] C. Höhne (for the NA49 collaboration), nucl-ex/0510049. Our present best fit (of the $4 \pi$ ratios) for the inhomogeneous model without independent fluctuations of $\mu_{S}$ yields $\bar{\Lambda} / \bar{p}=0.93$ at $E_{\mathrm{Lab}} / A=40 \mathrm{GeV}$, for example, versus 0.76 for $\delta T=\delta \mu_{B}=0$ (without contributions from weak decays).

[17] M. Gazdzicki et al. [NA49 Collaboration], J. Phys. G 30, S701 (2004) [arXiv:nucl-ex/0403023];

[18] J. Cleymans, B. Kämpfer, M. Kaneta, S. Wheaton, and N. Xu, Phys. Rev. C 71, 054901 (2005).

[19] O. Barannikova [STAR Collaboration], arXiv:nuclex/0403014; J. Adams et al. [STAR Collaboration], arXiv:nuclex/0501009; J. Adams et al. [STAR Collaboration], Phys. Rev. Lett. 92, 112301 (2004) [arXiv:nucl-ex/0310004];

[20] S. Eidelman et al. [Particle Data Group Collaboration], Phys. Lett. B 592, 1 (2004).

[21] For SPS-158 only the $4 \pi$ fit is shown; restricting the homogeneous fit to the mid-rapidity data gives smaller $\chi^{2} / \operatorname{dof}$, but still significantly higher than in the inhomogeneous approach. $\chi^{2}$ is smaller if other particle ratios are considered, as for example $\Xi / \Lambda, \Omega / \Xi$ instead of $\Xi / \pi, \Omega / \pi[6]$ or if finite widths of resonances are taken into account [9]. However, the increase of $\chi^{2}$ at SPS energies is generic if $\mathrm{Na} 494 \pi$-data are fitted.

[22] D. Zschiesche, arXiv:nucl-th/0505054, Fig. 7.

[23] C. Beck, Phys. Rev. Lett. 87, 180601 (2001); C. Beck and E. G. D. Cohen, Physica A 322, 267 (2003); H. Touchette and C. Beck, Phys. Rev. E 71, 016131 (2005). 